\title{
COMBINED GPR DATA ANALYSIS TECHNIQUE FOR DIAGNOSTICS OF STRUCTURES WITH THIN NEAR-SURFACE LAYERS
}

\author{
Dmitry O. BATRAKOV ${ }^{1}$, Angelika G. BATRAKOVA ${ }^{2}$, Mariya S. ANTYUFEYEVA ${ }^{1}$ \\ 1 - Department of Theoretical Radiophysics V. N. Karazin Kharkiv National University \\ Kharkiv, Ukraine \\ batrakov@karazin.ua, mariya.s.antyufeyeva@ieee.org \\ 2 - Chair of Highway Design, Geodesy and Land Management, Kharkiv National Automobile and Highway \\ University, Kharkiv, Ukraine \\ agbatr@ukr.net
}

\begin{abstract}
A method of processing GPR signals based on previously developed algorithms is proposed. The purpose of the study is to increase the accuracy of thickness measurements of near-surface thin layers of multilayer structures. The main idea of the method is to layer-by-layer restoration of physical and geometric characteristics of plane-layered media, for example, car coverings. As a result, with the help of numerical modelling, the operability and practical effectiveness of the proposed method was established. In addition, schemes for the practical implementation of this approach have been proposed for the processing of sounding data of pavement structures using a pulsed ground-penetrating radar "TRF-1". The use of additional procedures, for example, GPR calibration based on data obtained by core sampling, also improves the reliability and accuracy of assessing the current state of road pavements.
\end{abstract}

Keywords: non-destructive control, ground penetrating radar, road pavement.

\section{INTRODUCTION}

In many practical problems non-destructive testing of multilayer coatings that have thin nearsurface layers is needed $[1,2]$. By the term 'thin layers' we mean layers that thickness and other characteristic dimensions are commensurable or less than the resolving power of the diagnostic tool. The characteristic dimensions can be determined primarily by the physical properties of the materials, and in some situations by the dimensions of the surface irregularities [3]. In this case, the correct choice of diagnostic tools and data processing methods become particularly important. Universal methods and technical means for solving this problem for all possible cases do not currently exist. This is due to the peculiarities of the corresponding physical processes that are used in the diagnosis. Therefore, we will consider the solution of this problem with a concrete example control of the current state of the pavement of public roads. In the road surfaces, the upper layers (asphalt concrete) usually have a thickness of 4 up to $7 \mathrm{sm}$. The total thickness of the asphalt concrete can be up to $20 \mathrm{~cm}$ or more. The most effective tool for solving the problems of measuring the pavement layer thickness are georadars [4-7]. In addition to the possibility of non-destructive diagnostics, they have such important advantages as the ability to obtain and process data when the vehicle-laboratory moves with the speed of the traffic flow [8]. The diagnostic tool in the georadar application is the irradiated electromagnetic wave (most frequently Ultrawideband pulse - UWB). Carriers of useful information are waves reflected from inhomogeneities (external and internal boundaries, inclusions). The difficulties in solving the diagnostic problem in this case result from the presence of dispersion properties in electromagnetic waves. Difficulties are associated with the dependence of the attenuation of electromagnetic waves on the frequency and the electro physical properties of the layer materials. The problem is that the specific losses of the base layers (subgrade, base, subbase) and building materials of the upper layers are smaller for longer wavelengths, while the resolution is higher for waves with a shorter wavelength [9]. Sometimes two GPR are used to overcome these difficulties. One of antenna unit is placed in front of the laboratory car, and the other is placed behind the car or placed on the same cart [10]. In this case, the problem of their electromagnetic compatibility arises. This also complicates the use of so-called georadar lines for simultaneous scanning of the whole lane on the road $[11,12]$. Earlier, the authors presented a method of assessment the thickness of the road pavement layers, provided that the upper layer of the pavement has a small thickness (the optical path length in the material) [13]. It was pointed out that 
the measurement accuracy could be improved by using algorithms developed earlier. The key feature of the proposed method was the UWB signal and the introduction of a correcting factor in the calculation of the reflection coefficient from the outer and inner boundaries of the plane-layered medium.

However, the problem of developing a computational algorithm allowing the necessary correction automatically still remains unsolved. Therefore, the goal of this paper is to create an algorithm of processing GPR data and software, which allows accurate calibration in the real time to determine the true value of the layers thickness. Ultimately, the obtained results will create the basis of methods to improve the reliability of assessment the road pavement condition.

\section{THEORETICAL BACKGROUND AND THE PROBLEM STATEMENT}

The general problem of the current road pavement condition assessment is as follows. With the help of a UWB GPR, sensing of the pavement is carried out and then data is processed by the software "GeoVizy". The values of the layer thickness and the layer material relative permittivity
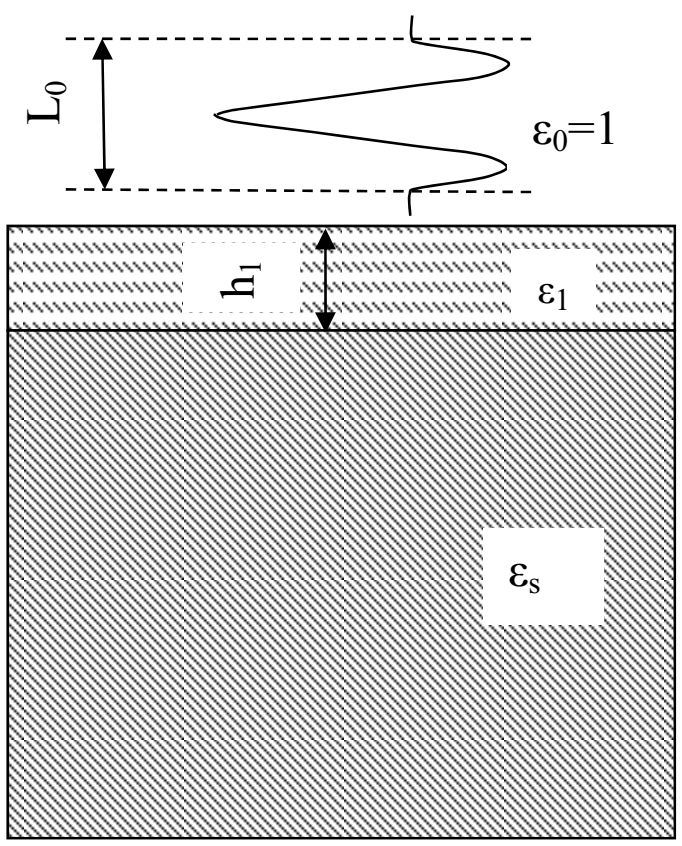

a)

Fig. 1. Model of the investigated medium (a) and the general view of the antenna unit $A B$ of the Georadar

Fig. 1. Model of the investigated medium (a) and the gener
«Odyag» (b) $(\varepsilon)$ are used as informative parameters evaluated applying reflected pulse signal processing. Data processing with the help of specialized programs allows to estimate the general pavement strength parameters. The analysis of the values $\varepsilon$ enables to assess the condition of the layer materials. This ultimately enables evaluation of the residual pavement lifetime. In [14] the general ideas of the normalization of reflected signals were briefly considered to study the possibility of increasing the accuracy and reliability of non-destructive testing with GPR. This work contains the development of these ideas, and their generalization to the case of other antenna unit. Fig. 1 explains statement of the problem. The ultra-wideband (UWB) probing pulse of the GPR falls normally to the outer surface of the studied plane-layer medium from the upper free half-space $\left(\varepsilon_{0}=1\right)$. The peculiarity of the problem in this case is that the spatial size of the pulse $L_{1}$ in the layer material $\left(L_{1}=c \cdot T / \sqrt{\varepsilon_{1}}\right)$ is larger or commensurate with the thickness of the layer. Figure 1.a presents one pavement layer ( $\left.h=h_{1}, \varepsilon=\varepsilon_{1}\right)$ and a semi-infinite base $\left(\varepsilon=\varepsilon_{S}\right)$ for simplicity. Figure 1.b shows the AB-1 antenna unit of the "Odyag" radar during remote sensing.

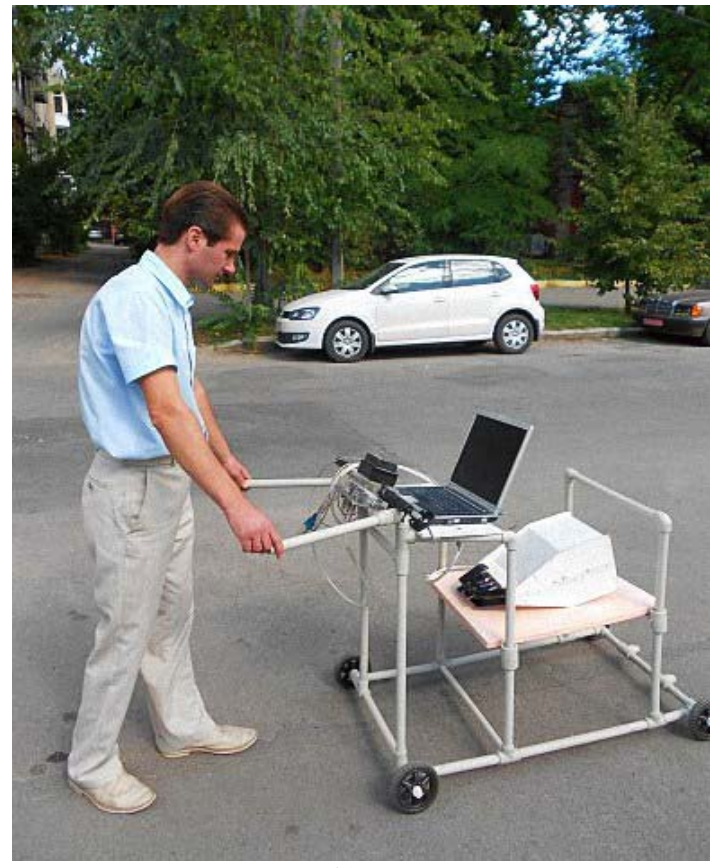

b)
The previously proposed data processing procedure $[6,8]$ is implicitly based on the assumption that the Fresnel formulas [14] for calculating the reflection and transmission coefficients of waves across the interface between two media are also valid for impulse signals. In this case (normal incidence) for the reflection $R_{n-1, n}$ and transmission $T_{n-1, n}$ coefficients of the wave incident on the layers boundary from the medium with the number $n-1$, will takes the form:

$$
R_{n-1, n}=\frac{\sqrt{\varepsilon_{n-1}}-\sqrt{\varepsilon_{n}}}{\sqrt{\varepsilon_{n-1}}+\sqrt{\varepsilon_{n}}},
$$




$$
T_{n-1, n}=\frac{2 \sqrt{\varepsilon_{n-1}}}{\sqrt{\varepsilon_{n-1}}+\sqrt{\varepsilon_{n}}} .
$$

According to the algorithm [6,8] formula (1) allows to calculate value $\varepsilon_{1}$ :

$$
\sqrt{\varepsilon_{1}}=\sqrt{\varepsilon_{0}} \frac{1-R_{0,1}}{1+R_{0,1}} .
$$

Before GPR data can be processed, a sensing signal must first be recorded. It can be done by laying a metal plate underneath the air launched antenna unit and having registration.

Since the reflection coefficient from the metal sheet is close to that $R \approx-1$, thanks to such a procedure, a pulse close to the probe pulse will be detected, but with a reversed polarity. After removing the metal sheet, reflection from the surface of the pavement is measured. The registration of these two signals allows us to calculate the reflection coefficient $R_{0,1}$ and using (3) to determine the $\varepsilon_{1}$. Then one can use (2) to calculate the amplitude of the wave incident on the lower boundary of the first layer and passing back through the outer boundary to the antenna together with the delay $\Delta t_{1}$ measurement, i.e. the time of passage of the layer in two directions enables to determine the thickness of the upper layer:

$$
h_{1}=\frac{\Delta t_{1} \cdot c}{2 \cdot \sqrt{\varepsilon_{1}}},
$$

where: $c$ is light speed in free space. Then the procedure is repeated for the next layer. In [6] it was introduced the transfer function of the system of $n$ layers. Such approach has the advantage of obtaining the transfer function of the $n$ layers medium by multiplying their own transfer functions $P_{n}$ :

$$
R_{n, \mathrm{n}+1}=A_{0}^{-1} \cdot C_{n} /\left(P_{1} \cdot P_{2} \cdot \ldots \cdot P_{n}\right),
$$

where: $P_{n}=\mathrm{T}_{n-1, n} \cdot \mathrm{T}_{n, n-1}$.

During the signal processing, it is necessary to have a method for determining the exact moment of the maximum signal to calculate its propagation time between reflections. To solve this problem, we used two methods: the Hilbert transform [15] and $(\mathrm{N}-1)$ times repeated integration of GPR signals [16] (we take $\mathrm{N}=2$ ).

The direct Hilbert transform

$$
\begin{aligned}
& \tilde{x}(t)=T H[x(t)]= \\
& =x(t) *(1 / \pi t)=\frac{1}{\pi} \int_{-\infty}^{\infty} \frac{x(\tau)}{t-\tau} d \tau
\end{aligned}
$$

for the arbitrary real function $x(t),(-\infty<t<\infty$ ) , could be given by convolution $x(t)$ with function $h b(t)=1 /(\pi t)$. Function $1 /(t-\tau)$ is called the kernel of the Hilbert transform. Integral of the transform has a singular point $a=t-\tau \Rightarrow 0$, in which one should use their Cauchy principal value: $\lim _{a \rightarrow 0}\left[\int_{-\infty}^{t-a} \ldots+\int_{t+a}^{\infty} \ldots\right]$. In the literature on the theory of signals Hilbert transform for analog signals is proposed to perform not according to the formulas of the linear convolution operator $1 / \pi t$, which leads to the $\infty$ at $t \Rightarrow 0$, but through a spectrum of the analytic function .

$$
\begin{aligned}
& z(t)=x(t)+i \widetilde{x}(t) \Leftrightarrow \\
& \Leftrightarrow X(v)+i \tilde{X}(v)=Z(v) ; \\
& i=\sqrt{-1 .}
\end{aligned}
$$

As for the repeated integration technique, unlike [16] we will use the one-times repeated integration procedure (OTRI). Obviously, in this case, at the "balance" points of the GPR pulse, its transformation will have roots (zeros). Points of "balance" are the moments of time when the area above the axis of abscissas is equal to the area of the signal under the abscissa. Fig. 2 shows the probing (1) and reflected (2) pulses. The medium consists of two layers of thickness $\mathrm{h} 1=4 \mathrm{sm}$ $\left(\varepsilon_{1}=6.5\right)$ and $\mathrm{h} 2=20 \mathrm{sm}\left(\varepsilon_{2}=4.3\right)$, which lie on a semi-infinite substrate with $\varepsilon_{s}=2.8$.

As one can see, the Hilbert transform shows two maxima (two boundaries) and OTRI-2 transformation curve has three roots, the curve crosses the axis three times (the studied medium is the same as in Fig. 2).

Next, we consider the real pulse signals of GPR. Fig. 4 shows the pulse of the antenna block $A B$ of the "Odyag" georadar (S) reflected from the metal sheet (a) and the signal (S) reflected from the road surface (b) together with the results of the Hilbert transform (1) and OTRI (2). As one can see, more than one maximum of the Hilbert transform (1) and several "sets" (R, $\left.r_{1}, r_{2}\right)$ of the OTRI (2) roots of transform correspond to the main signal, even when reflecting from the metal sheet.

One of the reasons of this result is the design of the antenna unit. The fact is that the differential antenna system using electric fields compensation to decouple the GPR antennas [6] is applied in the «Odyag» and «TRF-1» georadars. Antenna unit of the GPR "TRF-1" is shown in Fig.5. The UWB pulse is radiated by one transmitting dipole antenna, and the reflected signal is received by two receiving dipole antennas. 


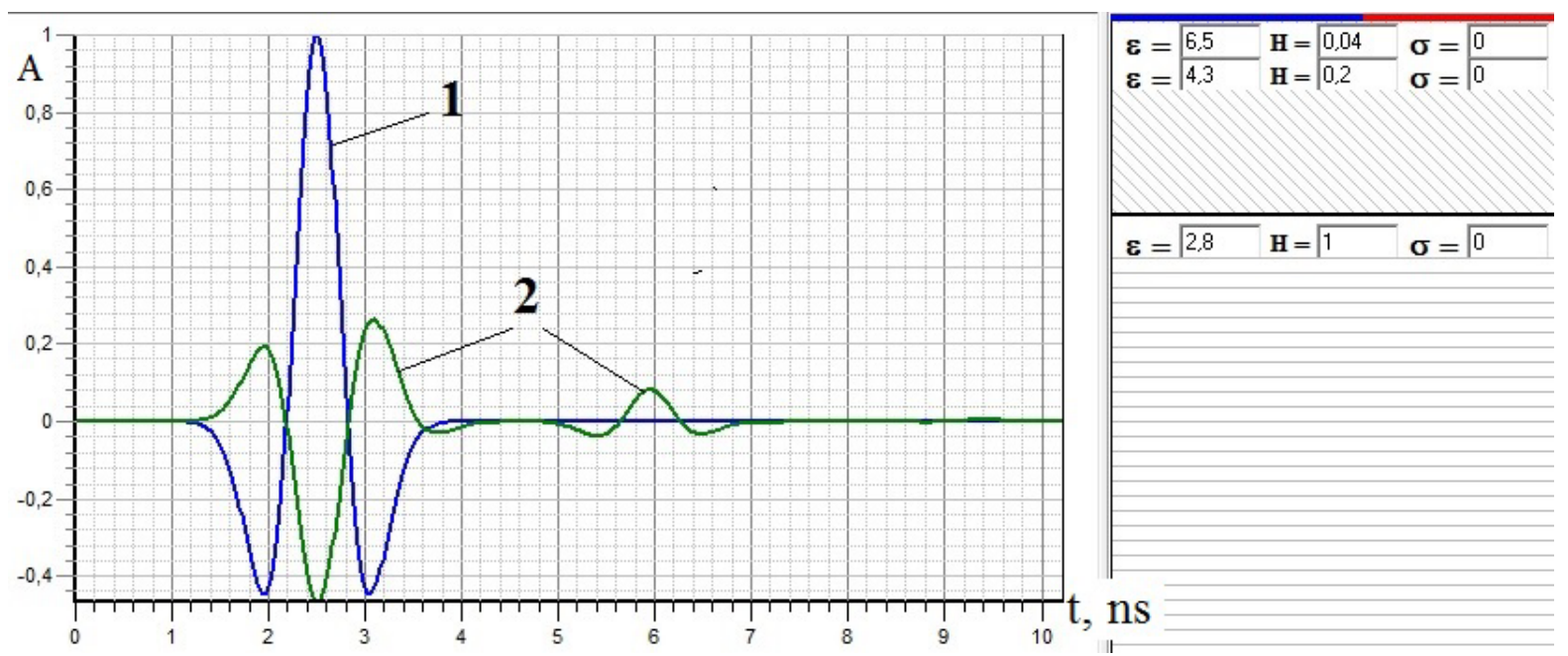

Fig. 2. The «probing» (1) pulse and pulse reflected from a two-layer medium on a semi-infinite substrate (2).

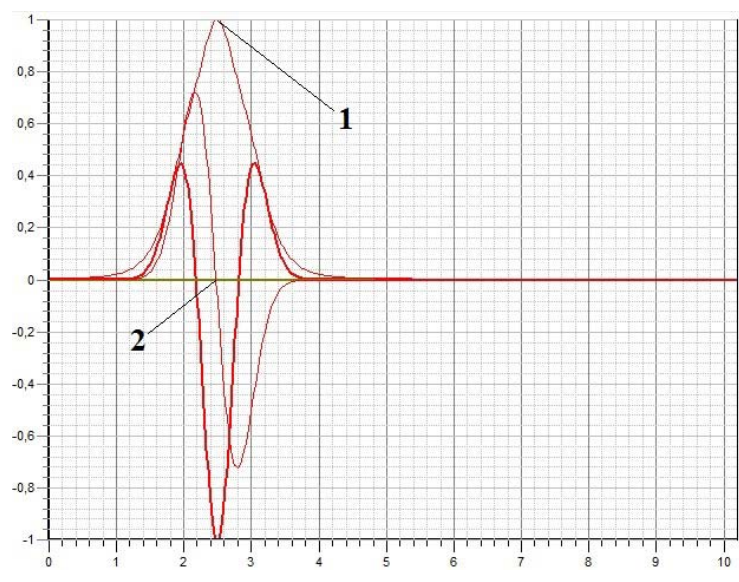

a)

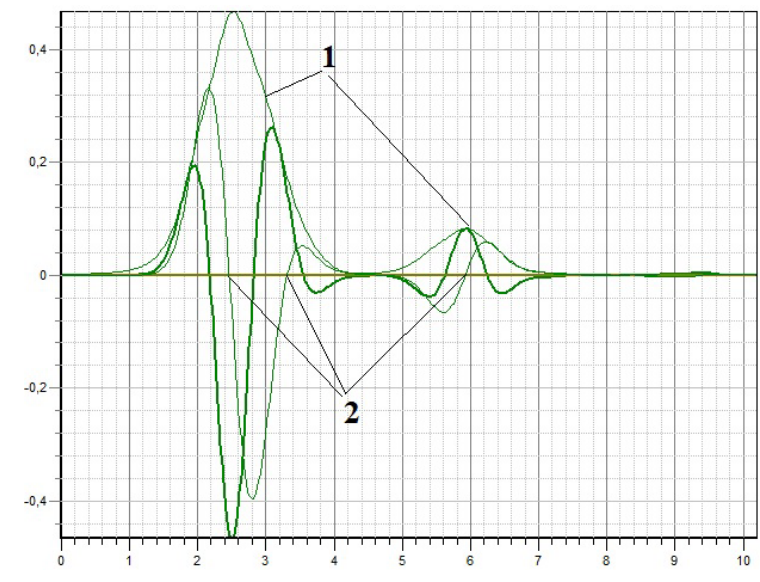

b)

Fig. 3. «Probing» (thick red curve (a)) and reflected (thick green curve (b)) pulses, as well as their Hilbert (1) and OTRI (2) transform curves.

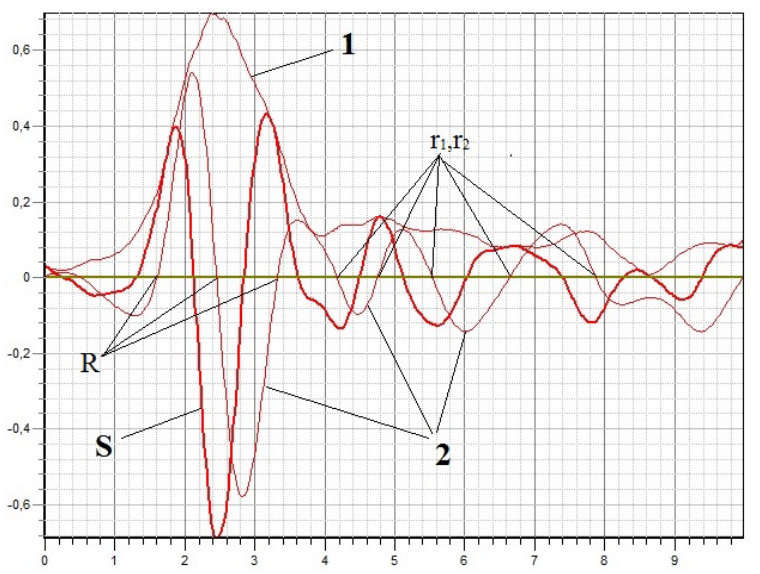

a)

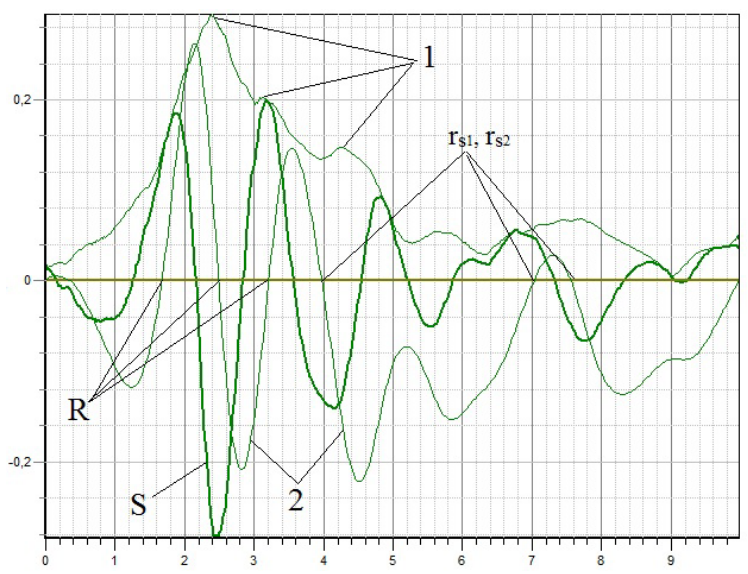

b)

Fig. 4. The pulse of the antenna unit $\mathrm{AB}$ of the "Odyag" radar (S) reflected from the metal sheet (a) and the signal (S) reflected from the road surface (b) together with the results of the Hilbert transform (1) and OTRI (2).
(a)- 20130418_113409_0 БC3_N12 Мет.txt
(b) - 20130418_113409_0 5C3_N18 Etalon.txt 


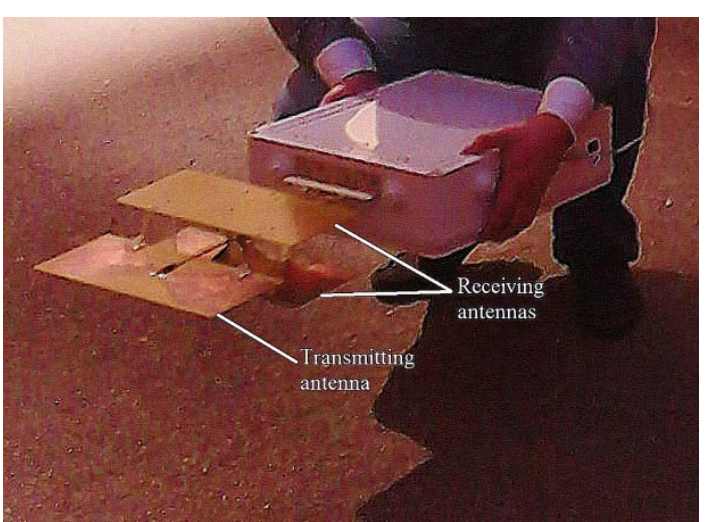

Fig. 5. The antenna unit of the GPR "TRF-1" during the sensing

The «arms» of these antennas are connected symmetrically (left bottom to right upper and vice versa) to ensure the symmetry of the receiving channel. The optimization of the distance between receiving antennas in this case is an independent problem, the solution of which was obtained earlier. On the basis of these data, it can be concluded that each return signal arriving at the receive path is a superposition of two signals shifted in time. This opens up additional possibilities for creating algorithms for processing sensing data.

Since achieving perfect symmetry between the receiving antennas has turned out impossible, the procedure of recording the feedthrough signal (transmitting antenna signal that has been received by the receiving antennas due to small symmetry violations between the receiving antennas) is used for a more accurate assessment of the layer thickness and the permittivity values of materials. This signal is recorded in absence of the objects near the antenna (Fig. 6). Then this signal is subtracted from the entire radargram and thus, we obtain a relatively clear signal reflected from the sensed structure.

In particular, a calibration point to the first root of the one-time repeated integration procedure can be automatically assigned to determine the value of the dielectric permittivity of the upper layer, but not the first maximum of the Hilbert transform [15]. For experimental verification of the proposed treatment method, a series of experiments were carried out on the reference structure. First of such constructions was brickwork of different thickness Fig.7.

During the experiments, the dielectric permittivity of the first layer have been determining in accordance with the formula (3), and further the layer thickness have been determining due to the specifying the delay $\Delta t_{1}$ and formula (4). Since in both considered cases (Fig. 7) this thickness was known, the results were compared with the actual ones. As a result, the error in recovering the thickness of the brick wall was $5 \%$ in the case of one layer $(12.5 \mathrm{~cm}$, fig. $7 \mathrm{a})$ and $6 \%$ in the case of two layers $(25 \mathrm{~cm}$, fig. $7 \mathrm{~b})$. It should be noted that the measurements were carried out only using the basic formulas $(3,4)$ and without additional correction according to $[7,10]$.

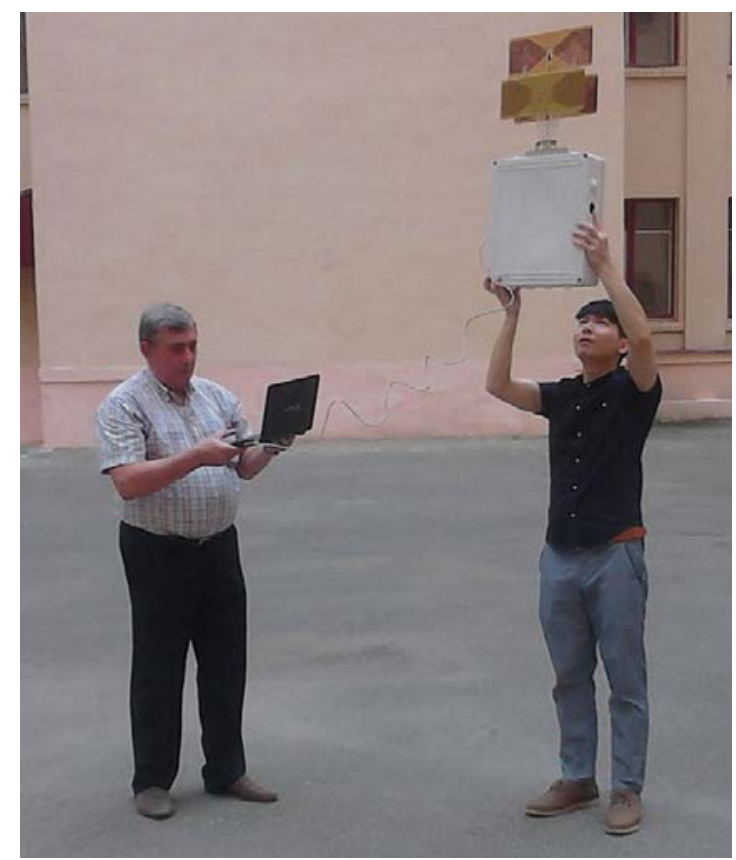

Fig. 6. The feedthrough signal recording

Also, experiments were carried out on structures with thin layers. An examples of some of these structures is shown in Fig.8, 9. As a result of numerous experiments and obtained data processing, it was found that when a thickness of the upper layer more than $10 \mathrm{sm}$. and a value $\varepsilon_{1}=6.0$ or more, the value $\varepsilon_{1}$ can be determined quite accurately without special treatment if the steepness of the front of the UWB probe pulse's main lobe is not more than $0.5 \mathrm{~ns}$. At the same time, if a thickness of the upper layer of $4 \mathrm{sm}$. $\left(\varepsilon_{1}=6.5\right)$, the standard processing algorithm used only Hilbert transform gives an inaccurate value of the dielectric permittivity. To solve this problem, computational experiments were carried out with the test pulses in the form of the second derivative of the Gauss function:

$$
\begin{aligned}
& S^{\prime \prime}(t)=\frac{d^{2}}{d t^{2}}\left(\exp \left(-\frac{t^{2}}{2}\right)\right)= \\
& =\left(t^{2}-1\right) \exp \left(-\frac{t^{2}}{2}\right)
\end{aligned}
$$


a)

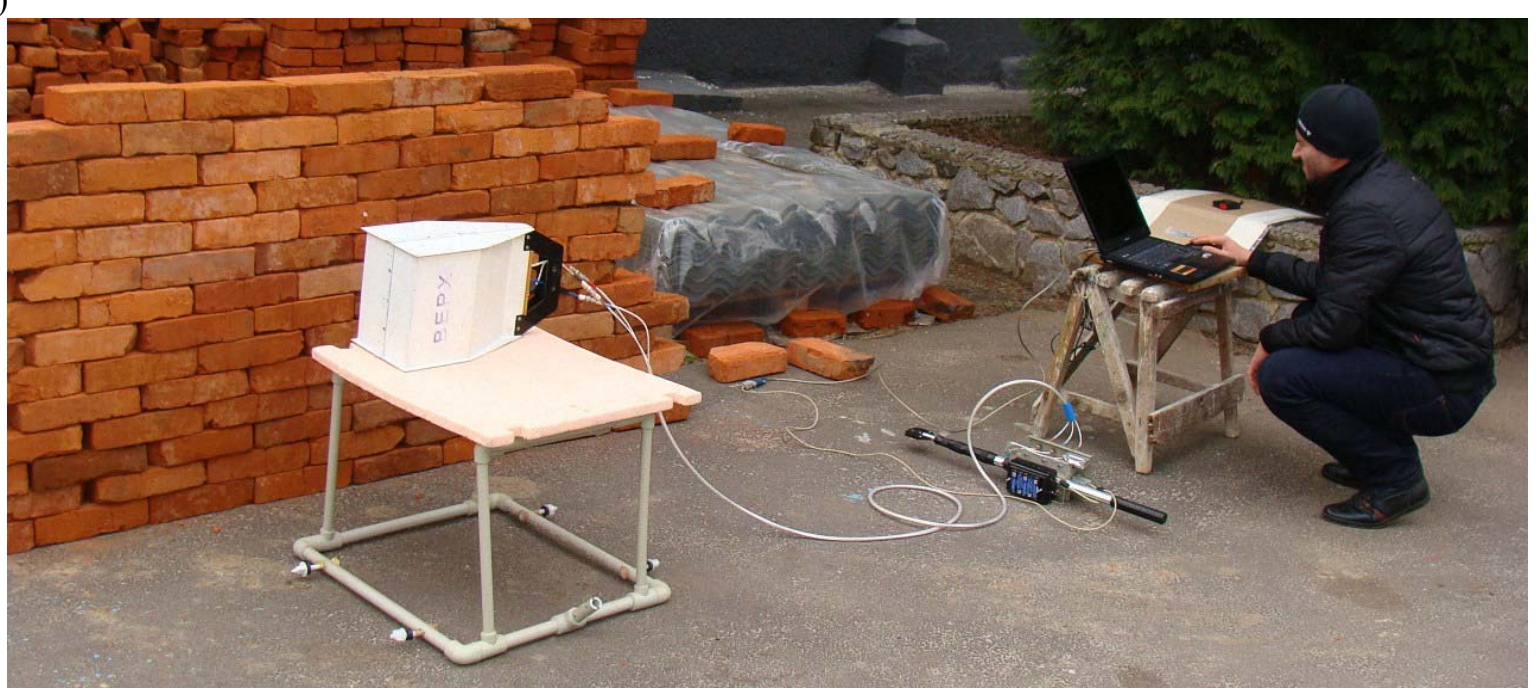

b)

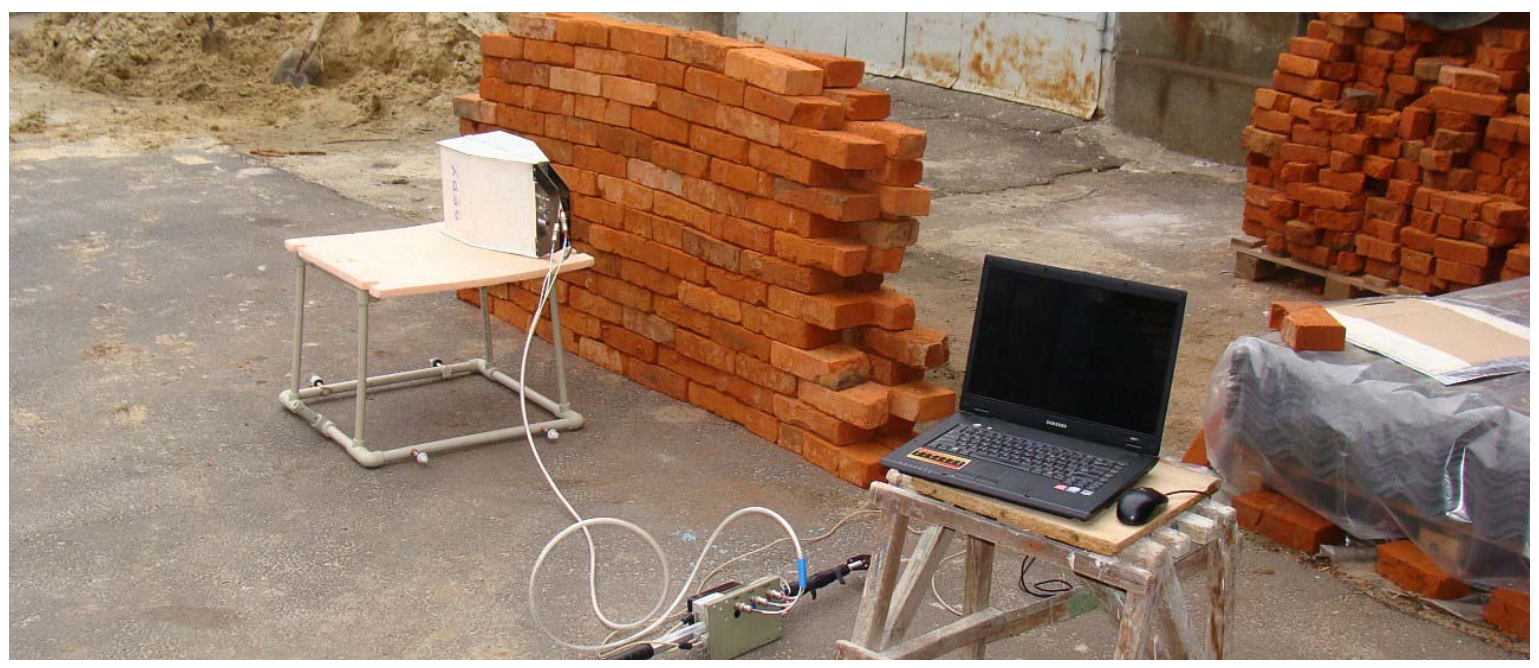

Fig. 7. Sensing of different model constructions for verification of the data processing algorithm

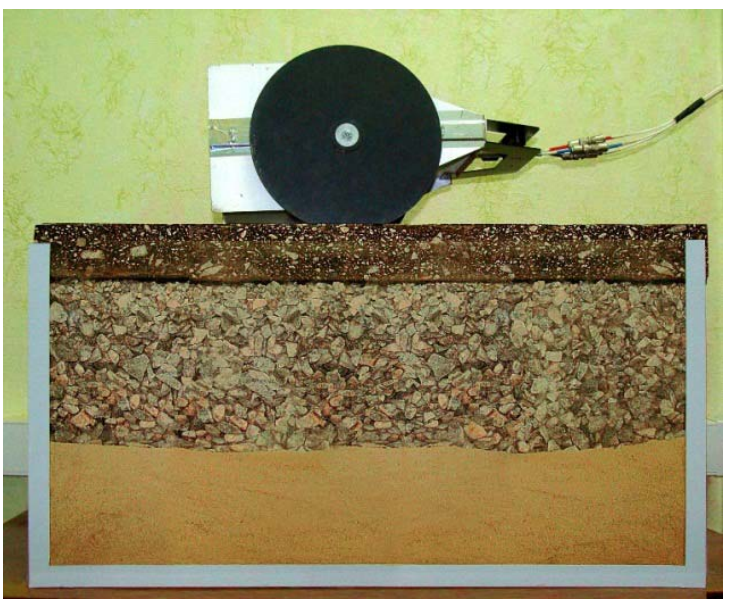

Fig. 8. Sensing of the structures with a thin top layer

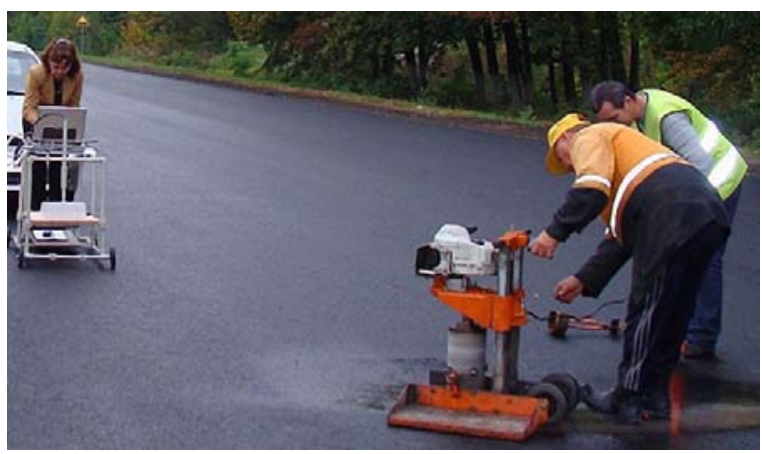

Fig. 9. Core sampling (right) while the received sensing data are processed (left)

Software "GeoVizy" was used for the simulation, because it allows not only to create virtual signals, but also to process them according to the algorithms proposed earlier. As an example, Fig. 8, 9 shows the results of the former semiautomatic mode and the new processing algorithm. Previously, when the "Min" button was pressed (step 1 in Fig. 10), the program automatically determined the first maximum of the Hilbert 
transform (for example, point 1 in Fig. 3a or "dt 0" in Fig. 10) and place (step 2 Fig. 10) a virtual cursor at this point on the time axis (point 2.4951 on the time axis in Fig. 8). Then (again in automatic mode), the reflection coefficient from the upper boundary was calculated and the value of the relative permittivity of the upper layer was determined from formula (3) (step 3 Fig. 10). In the second case (Fig. 9), according to the proposed algorithm, calibration (determination of the value of the reflection coefficient from the upper layer) is performed not on the first maximum of the Hilbert transform, but on the first maximum of the signal. This point in the case of the test signal defined by formula (8) can also be determined as the first root (zero) of the first derivative of the function describing the signal distribution in time. As can be seen, in the first case (Fig.10 is the standard processing), the value of the permittivity is determined with a great error $\varepsilon_{1}=7.55$ instead of the correct $\varepsilon_{1}=6.5$. The error in this case is (7.55$6.5) / 6.5 * 100=16,15 \%$. In the second case (fig. 11), the error of determining the permittivity is (6.46$6.5) / 6.5 * 100=0.61 \%$.

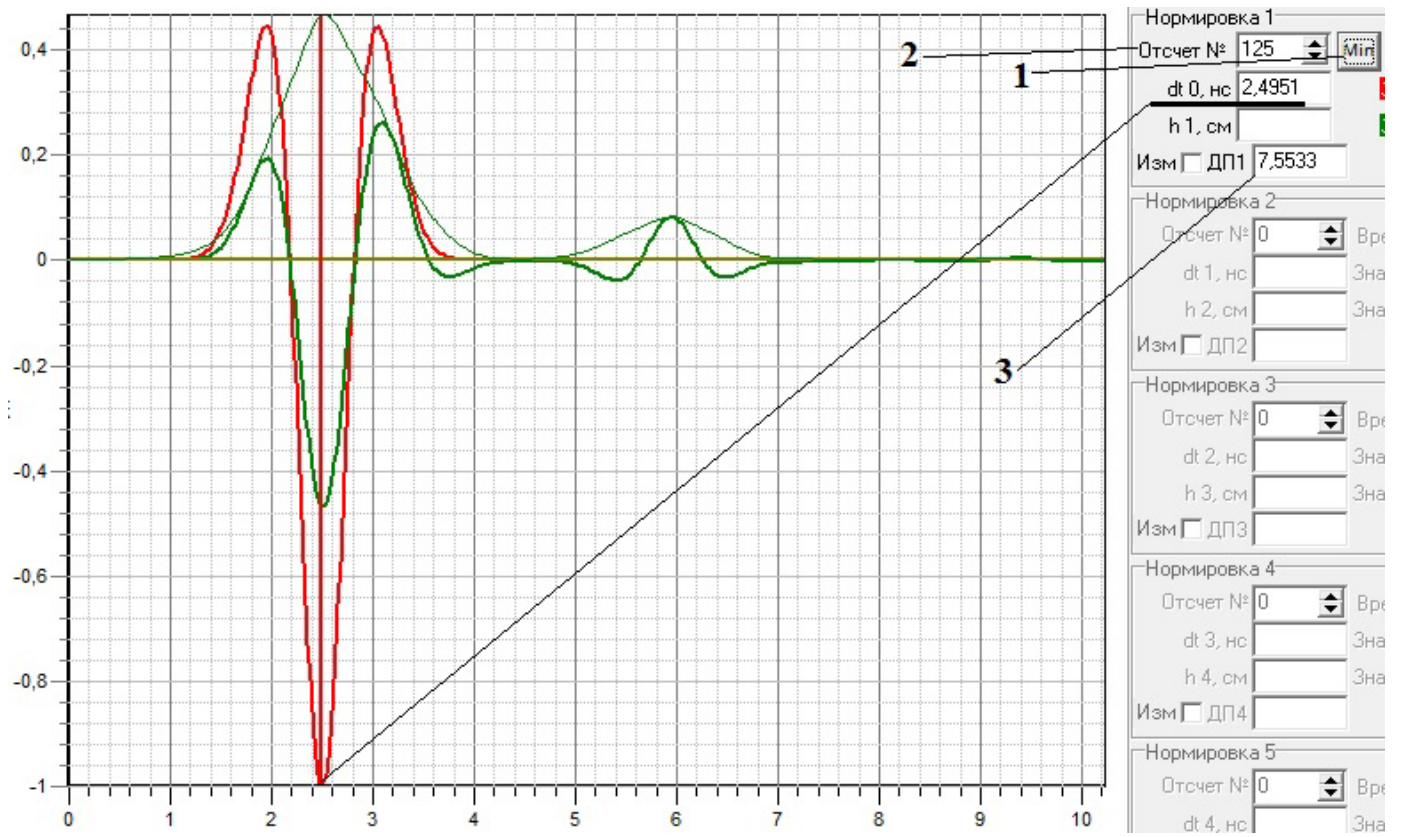

Fig. 10. Determination of the dielectric permittivity of the upper layer using the GeoVizy software (the former approach).

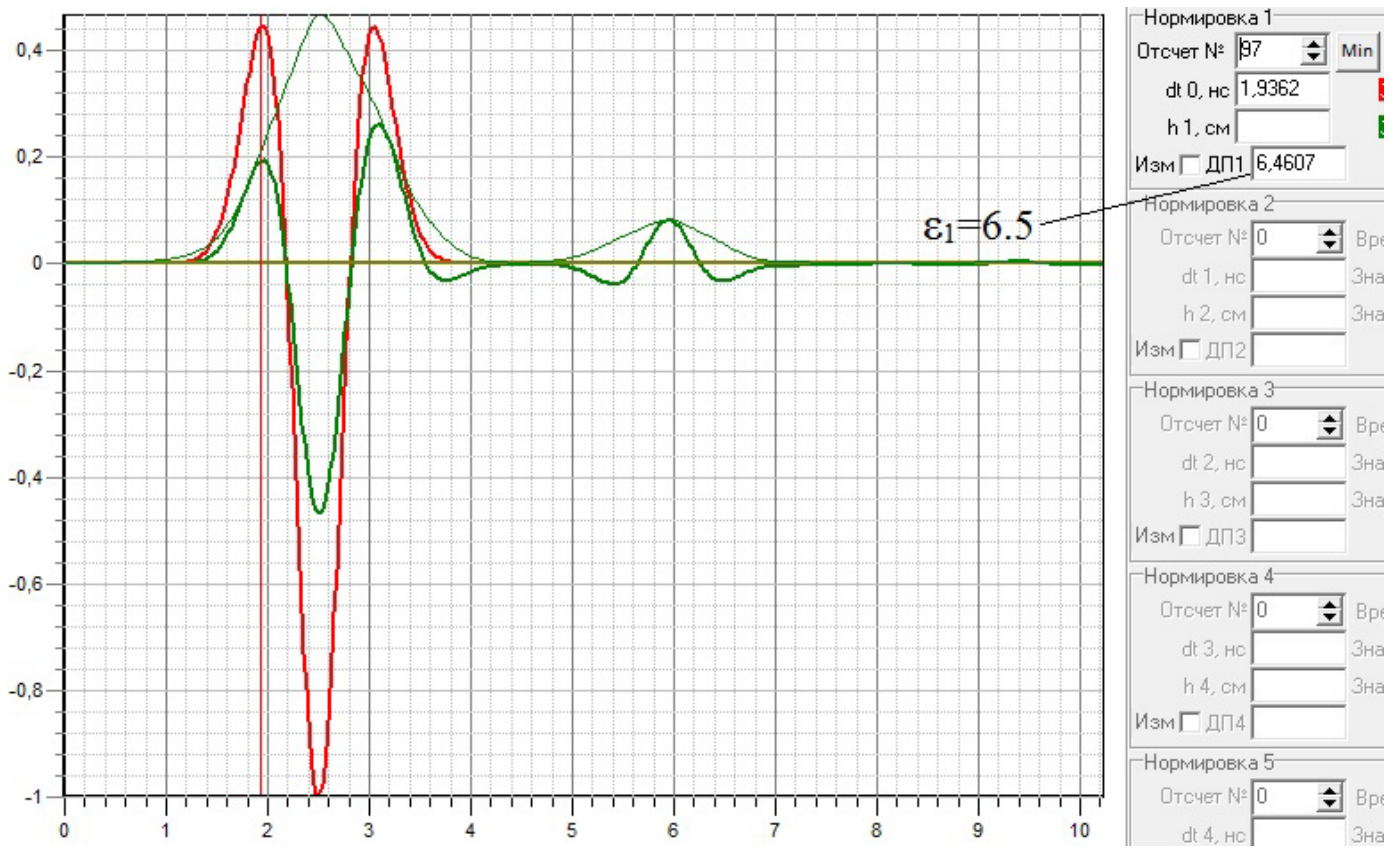

Fig.11. Determination of the dielectric permittivity of the upper layer using the GeoVizy software (proposed algorithm). 
The next stage of the GeoVizy program is for determination of the thickness of the upper layer and the permittivity of the second layer. To do this, one should to activate the virtual generation mode of the signal reflected from all the lower layers (marked as the "signal 3" on the "Signal construction" panel), except the first one (step 2) and then to activate the virtual generation mode of the transmitting antenna signal (step 3), which reached the second boundary (marked as the "signal 4 " on the "Signal construction" panel).

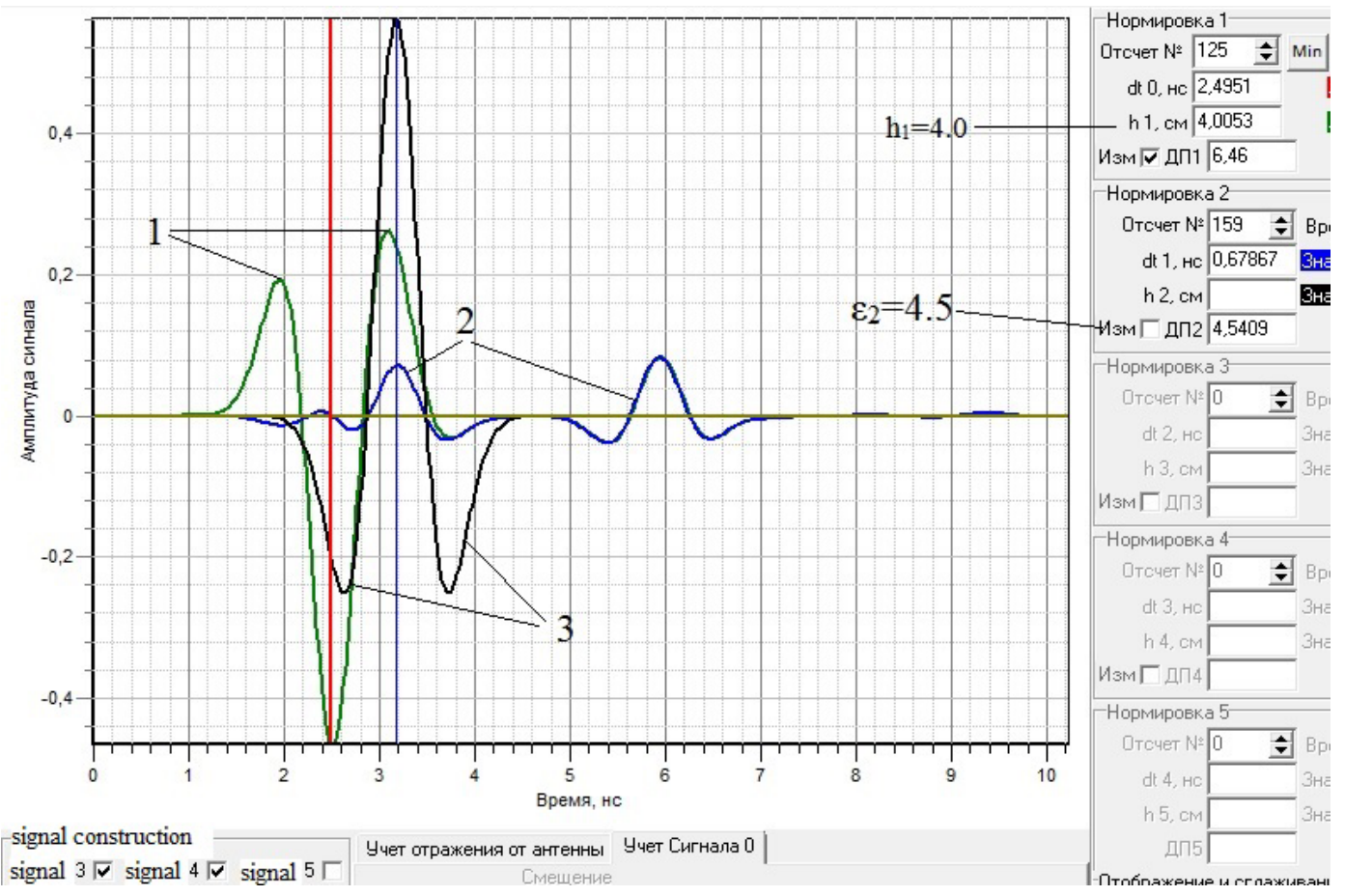

Fig. 12. Determination of the thickness of the upper layer

These signals are shown in Fig. 12 and marked with numbers 2 and 3, respectively. The thickness of the first layer under the standard processing algorithm is determined as $3.7 \mathrm{sm}$. (the error is (4$3.7) / 4.0 * 100=7.5 \%)$. In the second case, the determined thickness is $4.006 \mathrm{sm}$. (the error is $(4.006-4.0) / 4 * 100=0.15 \%$.). Thus, the error values of the key parameters recovered from the processing data differ in these two cases by more than 20 times. The value of the permittivity of the second layer is also automatically determined. The standard processing gives value $\varepsilon_{2}=5.24$, while the proposed processing algorithm provides the result $\varepsilon_{2}=4.5$. As can be seen, the error increases with processing, which is quite natural. In the standard approach of the processing, the error of the determination of $\varepsilon_{2}$ is $(5.24-4.3) / 4.3 * 100=21.86 \%$, but the error of the proposed algorithm is (4.5-3.3)/ $4.3 * 100=4.65 \%$. If necessary, it is possible to carry out further recovering of the dielectric permittivity values of the lower layers on the basis of the developed models (1) (5). It is clear that for the diagnosis of deeper layers, more advanced processing methods and technical means or a combination thereof are needed. This is due to both the accumulation of errors in the process of the multi-step recovery algorithm, and the difference between the real process of propagation of pulse signals and analogous processes within the framework of idealized models. The authors hope to present some results in this direction later.

\section{CONCLUSIONS}

UWB signals of pulsed georadars and appropriate methods of processing the obtained information are an effective tool for solving important applied problem of diagnostic and remote sensing. When assessing the current condition of road pavements using pulsed UWB GPR, a number of difficulties arise due to the presence of a small electrical thickness layers in the construction of the upper layers. Previously developed methods either give a big error, or require high qualification of the georadar operator and the use of additional information. An effective tool for increasing the accuracy of measurements is the proposed method for normalizing reflected signals with subsequent processing in accordance with previously developed algorithms. A key feature of the proposed approach is the correct choice of the normalization region of the UWB signal reflected from the medium and the introduction, if necessary, of a correction coefficient. This may be necessary to improve the accuracy of measuring the reflection coefficient from the outer and inner boundaries of a 
plane-layered medium. As a result based on the computational and laboratory experiments, the operability and effectiveness of the proposed algorithm have been demonstrated. Examples of the proposed approach application for data processing of pavement structure condition control using GPR are also given. The use of an additional calibration procedure based on the data obtained by core sampling also improves the reliability and accuracy of the assessment of the current road pavement condition. As directions for the further development of the proposed method and technical means, one should point out the possibility of improving the quality of signal processing by invoking methods for solving inverse problems for the sensing with different informative parameters [20-23], as well as expanding the capabilities of applications for the detection and positioning of various inhomogeneities [24-26].

\section{REFERENCES}

1. Parker F, Vecellio B, Greene J. Evaluation of the accuracy of pavement surface layer thickness measured with ground penetrating radar. Final report, project number 930-418, Auburn university highway research center, Auburn, Alabama, October, 1999.

2. Wissel T. Estimating soft tissue thickness from lighttissue interactions--a simulation study. Biomed / T Wissel, R Bruder, A Schweikard, F E. Opt Express. 2013 14;4(7):1176-87.

https://doi.org/10.1364/BOE.4.001176.

3. Zhuck NP, Batrakov DO. Determination of electrophysical properties of a layered structure with a stastistically rough surface via an inversion method. Physical Review B. - 1995;51(23):17073-17080.

4. Ground penetrating radar, theory and applications / [Jol Harry M. (Editor)]. - Amsterdam : Elsevier B.V., 2009.

5. Evans RD. Optimising ground penetrating radar (gpr) to assess pavements. A dissertation thesis submitted in partial fulfillment of the requirements for the award of the degree Doctor of Engineering (EngD), at Loughborough University, August 2009.

6. Pochanin GP, Masalov SA, Ruban VP, Kholod PV, Batrakov DO, Batrakova AG, Urdzik SN, Pochanin OG. Advances in short range distance and permittivity ground penetrating radar measurements for road surface surveying, in advanced ultrawideband radar: signals. Targets and Applications. 20-65. CRC Press - Taylor \& Francis Group, London. 2016.

7. Lachowicz J, Rucka M. Numerical modeling of GPR field in damage detection of a reinforced concrete footbridge. Diagnostyka. 2016;17(2):3-8

8. Batrakov DO, Antyufeyeva MS, Antyufeyev OV, Batrakova AG. GPR application for the road pavements surveys./ 2017 IEEE Microwaves, Radar and Remote Sensing Symposium (MRRS), 2017: 8184. http://ieeexplore.ieee.org/document/8075033/

9. Batrakov DO. Remote sensing of plane-layered media with losses using UWB signals. International Conference on Antenna Theory and Techniques (ICATT) Year: 2017:370-373 IEEE Conference
Publications.

http://ieeexplore.ieee.org/document/7972666/

10. Uzarowski L, Henderson V, Rizvi R, Mohammad K, Lakkavalli V. Use of FWD, GPR and IP in combination on complex pavement projects including case studies. Paper prepared for presentation. Conference of the Transportation Association of Canada, Toronto, 2016: 1-17.

11. Zehua Dong et al. Rapid detection methods for asphalt pavement thicknesses and defects by a vehicle-mounted ground penetrating radar (GPR) system. Sensors (Basel). 2016;16(12):2067. https://doi.org/10.3390/s16122067

12. Saarenketo T. Electrical properties of road materials and subgrade soils and the use of Ground Penetrating Radar in traffic infrastructure surveys Faculty of Science, Department of Geosciences, University of Oulu, Academic dissertation, 2006.

13. Batrakov DO, Antyufeyeva MS, Antyufeyev AV, Batrakova AG. UWB signal processing during thin layers thickness assessment. 2016 IEEE Radar Methods and Systems Workshop (RMSW)б 2016: $36-39$,

https://doi.org/10.1109/RMSW.2016.7778545

14. Born M, Wolf E. Principles of Optics (Sixth Edition). Pergamon, 1981.

15. Batrakov DO, Golovin DV, Simachev AA, Batrakova AG. Hilbert transform application to the impulse signal processing. 5th International Confernce, UWBUSIS' 2010:113-115. https://doi.org/10.1109/UWBUSIS.2010.5609110

16. Astanin LY, Kipke MV, Kostyleva VV. The structural features of ultrawideband signals // 2008 4th International Conference on Ultrawideband and Ultrashort Impulse Signals, 2008:180-182.

17. Batrakov DO, Tarasov MM. Algorithm of solving inverse scattering problems using the Pontryagin maximum principle. Radiotekhnika i Elektronika, 1999, 44(2):137-142.

18. Batrakov DO, Zhuk NP. Method for testing of layernon-homogeneous dielectrics using numerical solution of reverse problem dialing with dissipation in polarization parameters domain. Defektoskopiya (Russian Journal of Nondestructive Testing), 1994, 6: 82-87.

19. Batrakov DO, Batrakova AG, Golovin DV. Numerical simulation of UWB impulse response of plane layered media with 2D inclusion. 2012 6th International Conference on Ultrawideband and Ultrashort Impulse Signals 2012: 153-155.

20. Batrakov DO, Antyufeyeva MS, Antyufeyev A V, Batrakova AG. Remote sensing of plane-layered media with losses using UWB signals. International Conference on Antenna Theory and Techniques (ICATT), 2017:370-373 IEEE Conference Publications http://ieeexplore.ieee.org/document/7972666/

21. Batrakov DO, Antyufeyeva MS, Antyufeyev AV; Batrakova AG. GPR data processing for evaluation of the subsurface cracks in road pavements / 2017 9th International Workshop on Advanced Ground Penetrating Radar (IWAGPR), 2017:1-6, Edinbourg, Scotland, IEEE Conference Publications, http://ieeexplore.ieee.org/document/7996072/ 


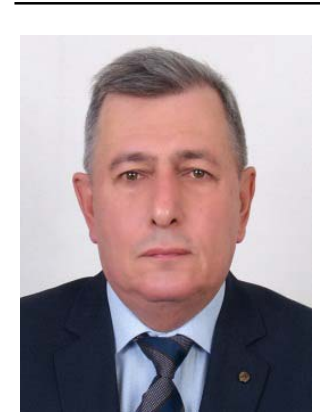

Dmitry O. BATRAKOV - Dr. Sci., Full Professor - Radiophysics, biomedical electronics and computer systems faculty, Chair of Theoretical Radiophysics, V. Karazin National University, Kharkiv, Ukraine, IEEE Senior member.

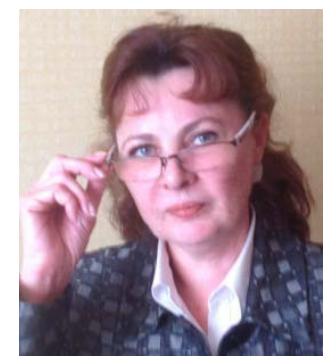

Angelika G. BATRAKOVADr. Sci., Professor - Chief of the Chair of Highway Design, Geodesy and Land Management, Kharkiv National Automobile and Highway University, Kharkiv, Ukraine.

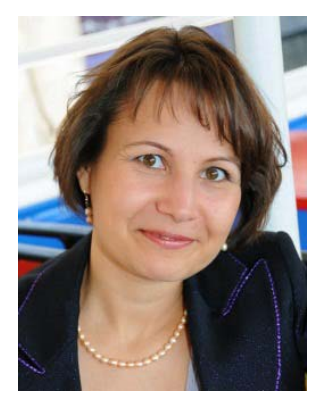

Mariya S. ANTYUFEYEVAPh.D., Senior Researcher Radiophysics biomedical electronics and computer systems faculty, Chair of Theoretical Radiophysics, V. Karazin National University Kharkiv, Ukraine. 